\section{OC-104 THE ENDOSCOPICALLY NORMAL COLON - WHEN IS MAPPING BIOPSY HISTOPATHOLOGICALLY JUSTIFIABLE?}

doi:10.1136/gut.2011.239301.104

V J Elliot," A C Bateman, ${ }^{1}$ B Green' Department of Cellular Pathology, Southampton University Hospitals NHS Trust, Southampton, UK

Introduction Mapping biopsy of the endoscopically normal colorectum is contentious ${ }^{1-3}$ and histopathological review of such biopsies is labour intensive. At a time when increasing efficiency and managing demand for pathological testing are key healthcare priorities, we aimed to identify situations where biopsy practice could be streamlined, without a detrimental impact on patients.

Methods Using our laboratory database, a search using the SNOMED system, retrieved cases coded as 'colonic mapping biopsy' over a 6 month period. The information was correlated with relevant endoscopy and pathology reports. The data were anonymised and entered on an Excel spreadsheet for analysis.

Results 360 cases were retrieved. 165 (46\%) were considered endoscopically normal/near normal. 151 (92\%) with endoscopically normal/near normal mucosa showed normal/near normal histology. 14 (8\%) endoscopically normal/near normal cases yielded a positive pathological diagnosis - melanosis coli (5), lymphocytic colitis (2), minimally active colitis (1), colitis unspecified (1), inflammatory bowel disease unspecified (1), inflammatory bowel disease of ulcerative colitis type (2), atrophy (1) and proctitis (1). Of the 360 cases studied, 164 $(46 \%)$ were referred with the clinical indication 'change in bowel habit' or 'colitis'. (The 69 cases referred to endoscopy with 'colitis' included approximately two thirds follow-up cases with a known diagnosis and around one third without a pre-existing diagnosis). The remaining 196 (54\%) cases were referred for various indications including iron deficiency anaemia and rectal bleeding. Positive diagnoses were yielded only from patients with 'change in bowel habit' or 'colitis'. No diagnoses were yielded from any other group.

Conclusion We have confirmed that pathological yield from biopsy of the endoscopically normal colon is highest in patients referred with 'change in bowel habit' or 'colitis'. We support biopsy in this cohort of individuals. However, mapping biopsy from endoscopically normal colonic mucosa in patients referred for other indications (eg, investigation of rectal bleeding or iron deficiency anaemia) should not be performed routinely as diagnostic yields are extremely low. Streamlining patients for biopsy on the basis of indication for referral would be a useful step in developing clinical protocols on when mapping biopsy of the endoscopically normal colon is histopathologically justifiable.

Competing interests None.

Keywords histopathology, mapping biopsy, normal colonoscopy

\section{REFERENCES}

1. Marshall JB, Singh R, Diaz-Arias AA. Chronic, unexplained diarrhea: are biopsies necessary if colonoscopy is normal? Am J Gastroenterol 1995;90:372-6.

2. Grant-Thompson W. Should a normal appearing colorectal mucosa be biopsied? Am J Gastroentero/ 1995;90:336.

3. Robinson RJ, Stone M, Mayberry JF. Normal rectal mucosa. Should we biopsy? Hepato-Gastroenterol 1997;44:703-5. 